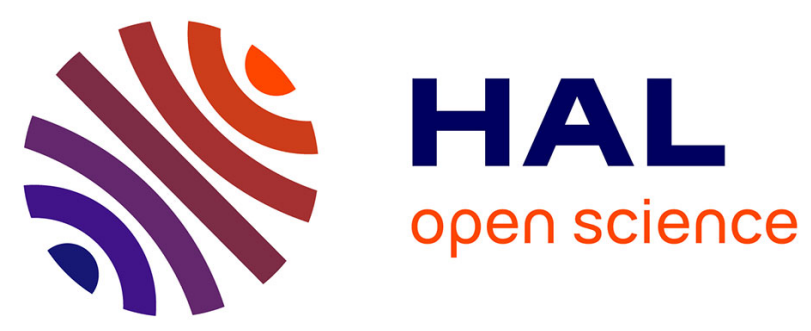

\title{
New online estimation algorithm of lateral tire-road coefficients based on Inertial Navigation System
}

\author{
Laetitia Li, Brigitte d'Andréa-Novel, Sylvain Thorel
}

\section{To cite this version:}

Laetitia Li, Brigitte d'Andréa-Novel, Sylvain Thorel. New online estimation algorithm of lateral tireroad coefficients based on Inertial Navigation System. 2019 IEEE Intelligent Transportation Systems Conference - ITSC, Oct 2019, Auckland, New Zealand. pp.3859-3866, 10.1109/ITSC.2019.8917532 . hal-02392141

HAL Id: hal-02392141

https: / hal-mines-paristech.archives-ouvertes.fr/hal-02392141

Submitted on 12 Dec 2019

HAL is a multi-disciplinary open access archive for the deposit and dissemination of scientific research documents, whether they are published or not. The documents may come from teaching and research institutions in France or abroad, or from public or private research centers.
L'archive ouverte pluridisciplinaire HAL, est destinée au dépôt et à la diffusion de documents scientifiques de niveau recherche, publiés ou non, émanant des établissements d'enseignement et de recherche français ou étrangers, des laboratoires publics ou privés. 


\title{
New online estimation algorithm of lateral tire-road coefficients based on Inertial Navigation System
}

\author{
Laëtitia Li ${ }^{1}$, Brigitte d'Andréa-Novel ${ }^{2,3}$ and Sylvain Thorel ${ }^{4}$
}

\begin{abstract}
For the sake of simplicity, control laws for autonomous vehicle mainly use linear tire models, but this modeling is only valid for small slip angles. Hence, to keep this hypothesis valid, tire's behavior has to lie within the limits of handling, i.e. there is a threshold the slip angle cannot surpass. This paper develops a new estimator for the cornering stiffness and maximum lateral friction coefficients. These parameters provide important information on the ground conditions and are beneficial for improving the stability of the vehicle. The algorithm is based on estimated lateral tire forces and on a 3 zones adaptive algorithm, including a Dugoff theoretical tire model. It will allow to set up a model not only for the linear part but for the whole range of slip angles, providing the trend of the tire behavior at each time and informing about the future evolution of lateral forces. The advantage of the algorithm is that no measurement of lateral tire forces is needed and few parameters are required such as yaw rate, longitudinal and lateral velocities obtained through an effective Inertial Navigation System, wheel rotational speeds and steering angles. Simulations conducted on realistic dynamical situation validate the algorithm efficiency.
\end{abstract}

\section{INTRODUCTION}

Control laws are mainly based on the assumption of linear tire model. Such an assumption is only valid for small slip angle and tire's behavior becomes highly nonlinear for severe manoeuvers, slippery road or at high-speed. To keep this assumption valid, tires slip angle must remain within the limits of linear tire's behavior which brings us to estimate the slip angle threshold $\beta_{t h}$ (see Fig.1). This information can be obtained through the knowledge of maximum lateral friction coefficient. The cornering stiffness $C_{\beta}$ is necessary in the context of closed loop in order to use the linear tire model. They are both important parameters of the tire/road interaction which depend mainly on the road conditions and are closely related to the tire efforts. Knowing precisely and in real-time these parameters allows to incorporate them in the control law and thus significantly impact the performance of the controller. As many factors affect the lateral friction coefficient and cornering stiffness, such as road surface conditions, tire types, temperature, tire pressure and so on, the identification of these parameters is still a challenging issue.

\footnotetext{
${ }^{1}$ Centre de Robotique, Mines ParisTech, PSL Research University, 60 boulevard Saint-Michel, 75272 Paris cedex 06, France laetitia.li@mines-paristech. fr

${ }^{2}$ Centre de Robotique, Mines ParisTech, PSL Research University, 60 boulevard Saint-Michel, 75272 Paris cedex 06, France brigitte. dandrea-novel@mines-paristech. fr

${ }^{3}$ STMS, UMR 9912-IRCAM-CNRS-SU, 1 place Igor Stravinsky, 75004 Paris, France brigitte.dandreanoveleircam. fr

${ }^{4}$ Safran Electronics \& Defense, 100 Avenue de Paris, 91344 Massy sylvain.thorelesafrangroup.com
}

Most of the approaches estimating the cornering stiffness use a linear adaptive tire model. For example, [1] and [2] estimate the cornering stiffness based on a gradient search algorithm and [3] uses a Kalman filter. Concerning tire-road friction coefficient, many researches have been done. Some of them try to estimate the parameters that affect the tireroad friction coefficient and try to predict the maximum friction coefficient $\mu_{\max }$ by using friction models. These methods require specific sensors (such as tire tread, optical or acoustic sensors) ([4], [5], [6]). In other approaches, researchers measure the effects of friction change on the tire behavior and developped slip-based $\mu_{\max }$ estimators [8]. The idea of slip-based estimator is to use the friction vs. slip data curve to identify $\mu_{\max }$. The slip-slope of this curve is related to a type of road and allows to establish a friction classifier. Some of the slip based $\mu_{\max }$ estimators work during braking [7], while other work during traction ([9],[10]). Slip-based approaches require a minimum of slip for the tire through acceleration, deceleration or steering maneuvers and have problem with calibration. In fact, the slip-slope used to classify the type of roads are sensitive to tire characteristics. Researchers also developed estimators based on theoretical model forces such as Burckhardt ([11],[12]) and LuGre ([13],[14]) models. These models are complex and the two parameters to be estimated are not clearly expressed. [15] and [16] use a brush tire model in which the two parameters clearly appear but only the friction coefficient is estimated. All these methods are based on a curve fitting approach and parametric identification to minimize the error between the developed force and the model-based force. To the best of our knowledge, [17] is the only paper estimating both the friction coefficient and the cornering stiffness. It uses a lateral tire model and the estimation is based on a parametric model and an adaptive law.

This paper concentrates on developing and demonstrating a reliable online algorithm for maximum lateral tire-road friction $\mu_{y_{\max }}$ and cornering stiffness $C_{\beta}$ estimation based on Inertial Navigation System measurements such as yaw rate, longitudinal and lateral velocities, as well as rotational speeds and steering angles. We also need a measure of wheel torque which could be given by an additional sensor or by the computed control law itself in a control loop context. The algorithm takes into account the linear and nonlinear part of tire characteristics by using a 3 zones adaptive algorithm. The inputs are the differences between estimated lateral forces and forces calculated with theoretical models: affine, Dugoff and constant. This algorithm includes a Levenberg Marquardt optimization method and a least squares fitting technique 


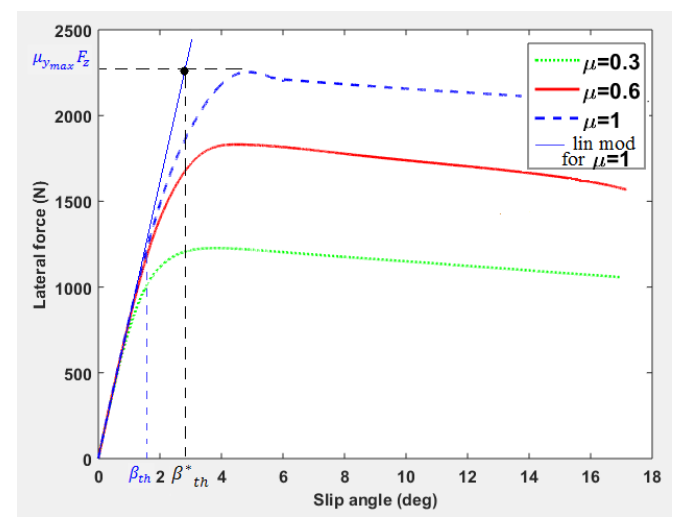

Fig. 1. Lateral force function of slip angle for various friction coefficients from experiments based on Pacejka model [30] (lin mod = linear model; $\beta_{t h}^{*}$ is obtained with (16))

with a sliding window as input.

The paper is organised as follows. In section II, the two parameters to be estimated and the Dugoff tire model are presented. In section III, all the inputs parameters for tire-road friction and cornering stiffness estimation are introduced and estimated, then the cornering stiffness and maximum lateral friction coefficient estimation algorithm is detailed in section IV. Section V shows the performance results of the estimator.

\section{MAXIMUM LATERAL FRICTION COEFFICIENT, CORNERING STIFFNESS AND DUGOFF MODEL}

The lateral force $F_{y}$ is mainly influenced by the slip angle $\beta$. This latter is defined as the angle between the direction of the wheel and the wheel speed vector. Fig. 1 shows this function $F_{y}(\beta)$. The slope at the origin is the so-called cornering stiffness $C_{\beta}$. As can be seen from Fig. 1, for small values of $\beta, F_{y}$ is proportional to $\beta$. For greater values of $\beta, F_{y}$ saturates. Vehicle dynamics depend on cornering stiffness $C_{\beta}$ and maximum friction coefficient $\mu_{y_{\max }}$ which are necessary to model the characteristics of tire/ground interaction. The limits of handling are defined from the maximum available lateral force

$$
F_{y_{\max }}=\mu_{y_{\max }} F_{z}
$$

where $F_{z}$ is the vertical tire force. Table I shows some typical value for $\mu_{y_{\max }}$ for different type of roads. The closer $\mu_{y_{\max }}$ is to 1 , the bigger $F_{y_{\max }}$.

TABLE I

SOME TYPICAL $\mu$ VALUES

\begin{tabular}{|l||c|c|c|c|}
\hline Road conditions & Asphalt dry & Asphalt wet & Snow & Ice \\
\hline Approximated $\mu$ & $0.9-1.0$ & $0.5-0.8$ & $0.2-0.3$ & $0.15-0.2$ \\
\hline
\end{tabular}

\section{A. Dugoff model}

There are many tire models that reflect the highly nonlinear relation between the lateral tire forces and slip angles.
These models range from a simple linear proportional model depending only on $C_{\beta}$ and $\beta$ to a nonlinear model such as the well-known Pacejka model, characterized by numerous empirical parameters [30]. The linear model has been widely used in many applications. This model is suitable for vehicles in normal driving situations where $\beta$ is low. However, as soon as the vehicle is subjected to severe maneuvers, $\beta$ becomes large and $F_{y}$ has a nonlinear behavior. So, a Dugoff model is chosen to describe the non linearities of tire's behavior with minimum complexity and qualitative correspondence with experimental behavior. It provides a simple formulation that can describe the forces under pure cornering, pure acceleration/braking and combined acceleration (braking)/cornering maneuvers. It assumes a uniform vertical pressure distribution on the tire contact patch.

Longitudinal force is expressed as $F_{x}=C_{\sigma} \frac{\sigma_{x}}{1+\sigma_{x}} f(\lambda)$ (see [33]) and lateral force as:

$$
F_{y}=C_{\beta} \frac{\tan (\beta)}{1+\sigma_{x}} f(\lambda)
$$

where $f(\lambda)$ is given by:

$$
\begin{aligned}
& f(\lambda)=\left\{\begin{array}{r}
(2-\lambda) \lambda \text { if } \lambda<1 \\
1 \text { if } \lambda \geq 1
\end{array}\right. \\
& \lambda=\frac{\mu_{\max } F_{z}\left(1+\sigma_{x}\right)}{2 \sqrt{\left(C_{\sigma} \sigma_{x}\right)^{2}+\left(C_{\beta} \tan (\beta)\right)^{2}}}
\end{aligned}
$$

with $\sigma_{x}$ the longitudinal slip ratio. These two equations depend on 4 important tire's parameters: the longitudinal stiffness $C_{\sigma}$, cornering stiffness $C_{\beta}$, normal force $F_{z}$ and maximum tire road friction coefficient $\mu_{\max }$.

Assuming pure slip conditions with negligible longitudinal slip, the simplified Dugoff model becomes:

$$
F_{y_{i j \mathrm{dug}}}=\left\{\begin{array}{l}
C_{\beta_{i j}} \tan \left(\beta_{i j}\right) \text { if } \lambda \geq 1 \\
C_{\beta_{i j}} \tan \left(\beta_{i j}\right)(2-\lambda) \lambda \text { if } \lambda<1
\end{array}\right.
$$

and $\lambda$ is given by:

$$
\lambda=\frac{\mu_{y_{i j} \max } F_{z_{i j}}}{2 C_{\beta_{i j}}\left|\tan \left(\beta_{i j}\right)\right|}
$$

The subscripts $i j$ here and in the sequel stand for $f l, f r, r l$ and $r r$ which respectively refer to front left, front right, rear left and rear right wheels. The tire force equations clearly show the two unknown parameters to estimate, the cornering stiffness $C_{\beta_{i j}}$ and the friction coefficient $\mu_{y_{i j} \max }$. This model synthesizes all the properties of the tire within these two parameters and this is the advantage compared to the Pacejka model, known as the reference model for the wheel/ground interaction modelling.

However, the accuracy decreases for large slip angles, compared to Pacejka's model. Indeed, when slip angles become large and enter into the nonlinear part, the forces obtained by the Dugoff model become larger than those computed by the Pacejka model. To briefly illustrate this, let us consider a vehicle moving on the trajectory illustrated in Fig. 6. Under normal driving conditions (lateral acceleration below $0.4 \mathrm{~g}$ ) as shown Fig. 2, on adherent ground like dry asphalt at $14 \mathrm{~m} / \mathrm{s}$ (full red and blue lines), Pacejka and Dugoff 


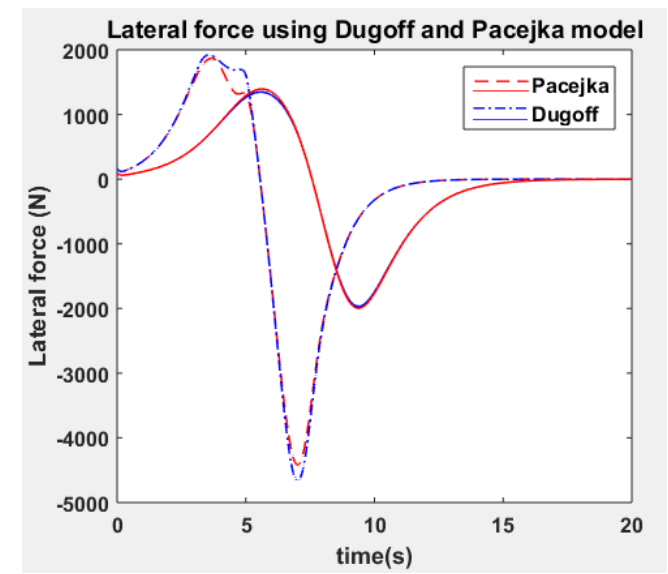

Fig. 2. Comparison of lateral forces with Dugoff and Pacejka tire model on dry asphalt for $v=19 \mathrm{~m} / \mathrm{s}$ (dotted line) and $v=14 \mathrm{~m} / \mathrm{s}$ (full line)

models fit very well. For $19 \mathrm{~m} / \mathrm{s}$ (dotted red and blue lines), as expected, we note that Dugoff's model slightly overestimates the lateral force when the lateral acceleration is high $(0.47 \mathrm{~g})$. Despite that, Dugoff model can fit correctly Pacejka model. Additionnaly, as already mentioned in the introduction, the algorithm for the estimation of $\mu_{y_{i j} \text { max }}$ and $C_{\beta_{i j}}$ is separated into 3 zones and when Dugoff model becomes incorrect, a constant model is used, as it will be detailed in section IV.

\section{PARAMETERS ESTIMATION}

In our estimation algorithm, in order to use Dugoff model, slip angle, vertical forces, longitudinal and lateral forces have to be estimated. This is the subject of this section.

\section{A. Slip angles estimation}

Tire slip angle can be calculated as:

$$
\begin{aligned}
& \beta_{f l}=\delta_{f l}-\arctan \left(\frac{V_{y}+l_{f} \dot{\psi}}{V_{x}-\frac{e_{f}}{2} \dot{\psi}}\right) \\
& \beta_{f r}=\delta_{f r}-\arctan \left(\frac{V_{y}+l_{f} \dot{\psi}}{V_{x}+\frac{e_{f}}{2} \dot{\psi}}\right) \\
& \beta_{r l}=\delta_{r l}-\arctan \left(\frac{V_{y}-l_{r} \dot{\psi}}{V_{x}-\frac{e_{r}}{2} \dot{\psi}}\right) \\
& \beta_{r r}=\delta_{r r}-\arctan \left(\frac{V_{y}-l_{r} \dot{\psi}}{V_{x}+\frac{e_{r}}{2} \dot{\psi}}\right)
\end{aligned}
$$

where $l_{f}$ and $l_{r}$ are respectively the distance between the center of gravity and the front axle and the center of gravity and the rear axle, $e_{f}$ and $e_{r}$ are the front and rear tracks, $V_{x}$ and $V_{y}$ are the longitudinal and lateral speed of the center of gravity, $\dot{\psi}$ the yaw rate and $\delta_{i j}$ the steering angles.

\section{B. Normal tire force estimation}

One way to estimate the vertical forces $F_{z_{i j}}$ is based on wheel suspension dynamics [18], but it needs sensors like suspension deflection sensors which are expensive and not present on our vehicle. Thus, we will estimate the static vertical forces, i.e. ignoring suspensions dynamics, based on vehicle weight, center of gravity position, vehicle longitudinal and lateral acceleration.

Under normal driving conditions, the vertical forces are mainly due to the gravity. During acceleration, braking or bending phases, vertical forces vary due to inertial forces (load transfer). By neglecting the coupling between pitch and roll dynamics, the vertical forces are [18]:

$$
\begin{aligned}
F_{z_{f l}} & =m \frac{l_{r}}{2 l} g-m \frac{h a_{x}}{2 l}-m \frac{h a_{y} l_{r}}{e_{f} l} \\
F_{z_{f r}} & =m \frac{l_{f}}{2 l} g-m \frac{h a_{x}}{2 l}+m \frac{h a_{y} l_{r}}{e_{f} l} \\
F_{z_{r l}} & =m \frac{l_{f}}{2 l} g+m \frac{h a_{x}}{2 l}-m \frac{h a_{y} l_{f}}{e_{f} l} \\
F_{z_{r r}} & =m \frac{l_{f}}{2 l} g+m \frac{h a_{x}}{2 l}+m \frac{h a_{y} l_{f}}{e_{f} l}
\end{aligned}
$$

where $m$ is the vehicle mass, $g$ the gravity acceleration, $h$ the height of the center of gravity, $a_{x}$ and $a_{y}$ the longitudinal and lateral acceleration at the center of gravity.

\section{Longitudinal tire force estimation}

Longitudinal tire forces in tire frame $F_{x p_{i j}}$ can be calculated from the wheel rotation dynamics:

$$
F_{x p_{i j}}=\frac{T_{\omega_{i j}}-I_{r} \dot{\omega}_{i j}}{r_{i j}}
$$

where $T_{\omega_{i j}}$ is the wheel torque, $I_{r}$ the wheel moment of inertia, $\dot{\omega}_{i j}$ the angular velocity and $r_{i j}$ the effective radius of the wheel. Wheel torques are the control variables which are computed if the estimation is integrated into a control loop otherwise torque sensors are needed.

\section{Lateral tire force estimation}

The problem of estimating lateral tire forces $F_{y}$ has been extensively studied in the dynamic vehicle community. Some methods use tire models to estimate axle per axle lateral forces by neglecting rear longitudinal forces ([19],[20]). Other methods are also based on tire models but use a 4 wheels model and estimate the forces via an EKF ([21],[22],[23]) or UKF [24].

In our case, we do not want to use a force model to estimate $F_{y}$ because a force model is already used to estimate $C_{\beta_{i j}}$ and $\mu_{y_{i j} \max }$. In addition, not using a complex force model can save computing time and increase robustness compared to variations in road conditions. For example, ([25],[26],[27],[28]) do not use a tire model but neglect rear longitudinal tire forces and estimate lateral tire force per axle thanks to an observer. [29] estimates the lateral tire forces witout using tire model but based on yaw moment calculation $M_{G}$ at the center of gravity $G$ and $M_{G_{i j}}$ at the center of each wheel $G_{i j}$, leading to the following equations :

$$
\left\{\begin{array}{l}
M_{G}=I_{z} \ddot{\psi}=T_{x_{0}}+T_{y_{0}} \\
M_{G_{i j}}=M_{G}+m\left(\overrightarrow{a_{G}} \wedge \overrightarrow{G G_{i j}}\right) \cdot \overrightarrow{z_{G}}=T_{x_{i j}}+T_{y_{i j}}
\end{array}\right.
$$


where $\overrightarrow{a_{G}}$ is the acceleration vector at $G, \overrightarrow{z_{G}}$ the vertical axis attached to the vehicle frame and $T_{x}$ and $T_{y_{\bullet}}$ respectively include the terms depending on longitudinal and lateral tires forces (see [29]). By adapting [29] to four steering wheels vehicle and lumping (9) as a matrix form gives :

$$
\begin{aligned}
& \hat{F_{Y}}=H^{-1} T_{Y}
\end{aligned}
$$

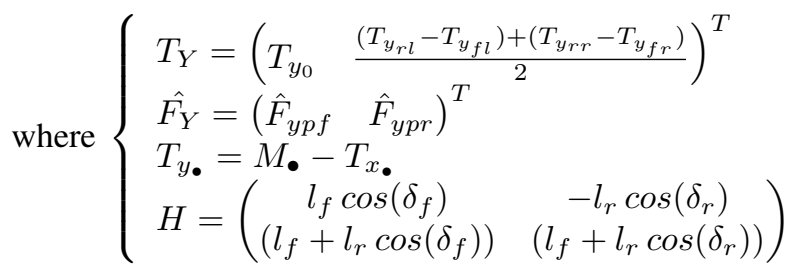

where $F_{y p f}$ and $F_{y p r}$ stands for the front and rear total lateral force in the tire frame. (10) gives the estimation of front and rear lateral forces per axle and then lateral forces at each wheel are calculated from a vertical force distribution:

$$
\begin{array}{ll}
\hat{F}_{y p f l}=\frac{F_{z f l}}{F_{z f l}+F_{z f r}} \hat{F}_{y p f} & \hat{F}_{y p f r}=\frac{F_{z f r}}{F_{z f f l}+F_{z f r}} \hat{F}_{y p f} \\
\hat{F}_{y p r l}=\frac{F_{z r l}}{F_{z r l}+F_{z r r}} \hat{F}_{y p r} & \hat{F}_{y p r r}=\frac{F_{z r r}}{F_{z r l}+F_{z r r}} \hat{F}_{y p r}
\end{array}
$$

In what follows, we call the measured lateral force, the force obtained in simulation with the Pacejka model and the estimated lateral tire forces the ones obtained with (8)(11). For a lane-change trajectory such as the one depicted in Fig. 6 at 18m/s, Fig. 3 and Fig. 4 respectively show the estimated and measured front axle lateral force and the estimated and measured lateral tire force for the front left wheel. As depicted Fig. 3, the error is low but reaches $20 \%$ when the vehicle takes the corner with a lateral acceleration of $0.4 \mathrm{~g}$. Concerning the case shown in Fig. 4 the error reaches $35 \%$ during the turn. Thus, it can be noted that the estimation is quite relevant even for high lateral acceleration.

\section{ESTIMATION ALGORITHM OF MAXIMUM LATERAL FRICTION AND CORNERING STIFFNESS}

By studying $F_{y}(\beta)$ curve in Fig. 5, we divide the curve into 3 zones : linear, transitional and nonlinear regimes as depicted in red. For the estimation purpose, we also distinguish 3 others areas of estimation :

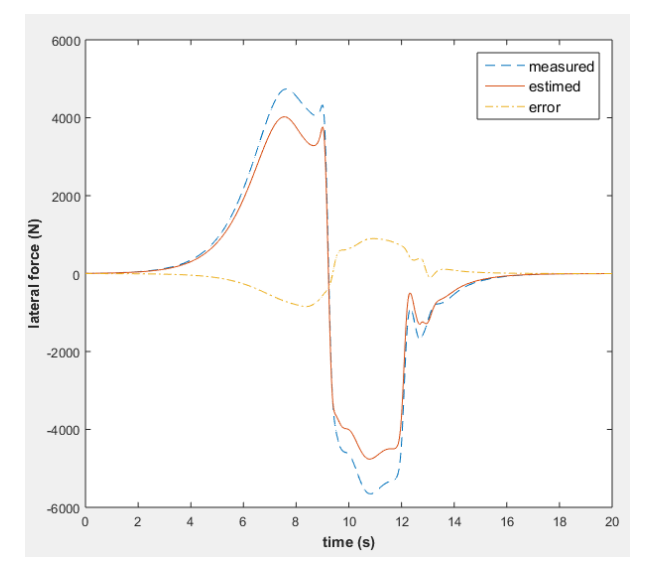

Fig. 3. Front axle total lateral forces

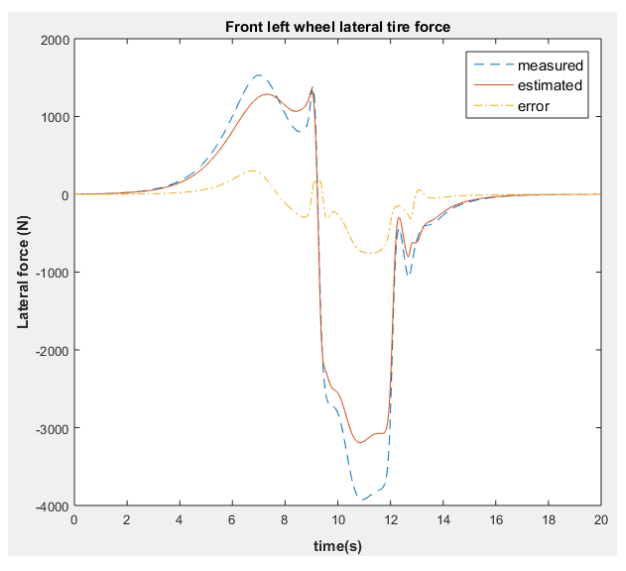

Fig. 4. Front left lateral tire force

- an affine zone belonging to the linear regime where $F_{y_{i j}}^{*}=a_{1} \beta_{i j}+b_{1}$

- a Dugoff model zone overlapping the transitional regime and a part of the linear and nonlinear regime, where $F_{y_{i j}}^{*}=F_{y_{i j \mathrm{dug}}}($ see (2)) ;

- a constant zone belonging to the nonlinear regime where $F_{y_{i j}}^{*}=b_{2}$

The delimitation of these estimation areas differs from the one usually defined for the tire model regions as depicted in green in Fig. 5. The aim is to find $C_{\beta_{i j}}$ and $\mu_{y_{i j} \max }$ that minimize the error between the lateral force $F_{y_{i} \text { provided by }}^{*}$ the 3 different theoretical models mentioned above and the one estimated with (8)-(11), $\hat{F}_{y p i j}$. For the affine zone, the linear least squares fitting technique is applied, for the Dugoff model zone, the Levenberg Marquardt (LM) algorithm is used and for the constant zone, the constant $b_{2}$ is chosen equal to the maximum lateral tire force of the window.

Splitting the estimation into several zones allows us to limit the range where the Dugoff model is applied to prevent from possible errors and wrong convergence due to the $\mathrm{LM}$ algorithm. When $\beta$ is very small, lateral forces can be approximated by an affine model. This area is framed by the y-intercept values $b_{1}$. In the nonlinear region, the constant model is applied. The transition between the different zones is function of the slope $a_{1}$ and the y-intercept values $b_{1}$.

As input for the algorithm, a sliding window is used. It contains a set of $\left\{\hat{F}_{y_{i j}}(k), \beta_{i j}(k), t(k)\right\}$ data, with $1 \leq k \leq$ $N$. $t$ is the time and $N$ the empirically determined window's maximum length. Accumulating values over time will avoid to obtain aberrations in the estimation. Trade-off must be made since a large data set causes delays and increases the computational load but it achieves better performance. This window, with variable length, will depends on spatial and temporal factors. With $\epsilon_{1}, \epsilon_{2}, \tau_{1}$ and $\tau_{2}$ which are thresholds fixed by the user, the window is constructed as follows :

- a data is added to the window if :

- the difference between the last slip angle $\beta(k)$ of the window and the current one $\beta(k+1)$ is greater than $\epsilon_{1}: \quad|\beta(k+1)-\beta(k)| \geq \epsilon_{1} \rightarrow$ add data

- there was no data added for a long time : 


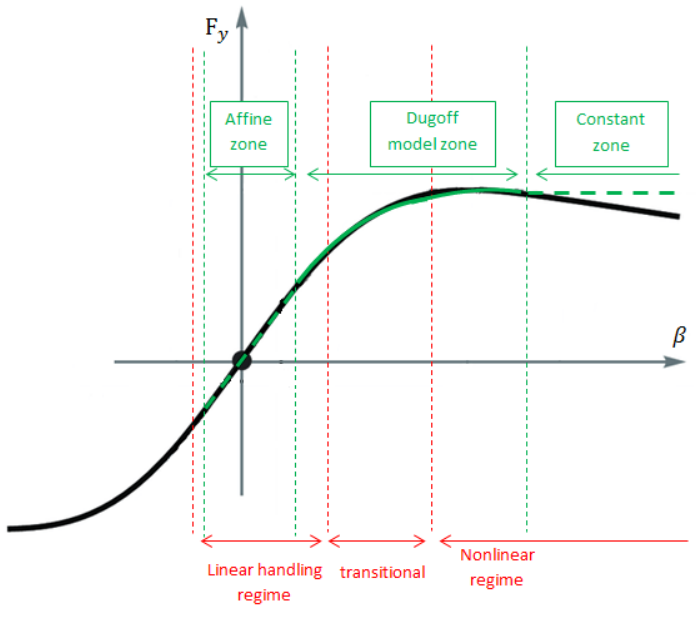

Fig. 5. Tires different regimes and zones for estimation

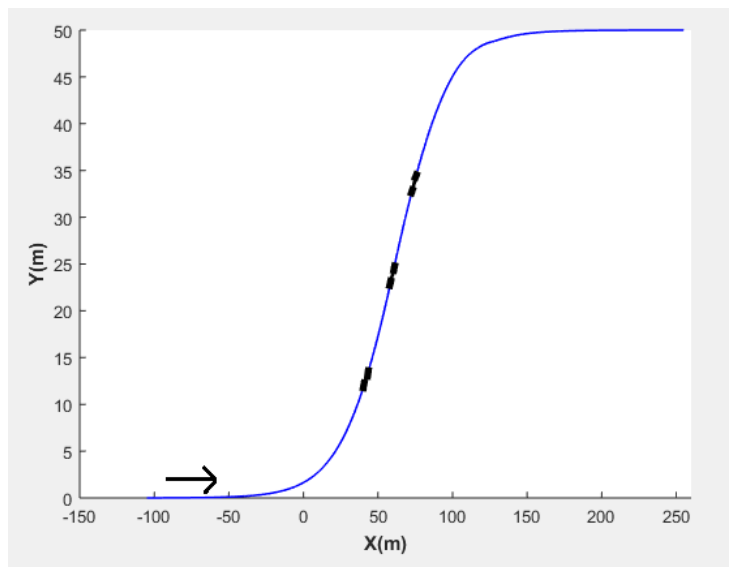

Fig. 6. Lane-change trajectory

$$
|t(k+1)-t(k)| \geq \tau_{1} \rightarrow \text { add data }
$$

- the window is refreshed if :

- the difference between the first slip angle $\beta(1)$ of the window and the last one $\beta(k)$ is greater than $\epsilon_{2}:|\beta(1)-\beta(k)| \geq \epsilon_{2} \rightarrow$ delete first data

- a data is in the window for a long time : $|t(1)-t(k)| \geq \tau_{2} \rightarrow$ delete first data

- the window is full : delete one out of two data

\section{A. Affine zone}

Why not using the Dugoff in the entire linear regime? The LM algorithm takes as input partial derivatives w.r.t the parameters we want to estimate (12). One may note that if the $\lambda$ parameter in the Dugoff model (2) is such as $\lambda \geq 1$, i.e the tire is in the linear part, $\mu_{y_{i j} \text { max }}$ cannot be estimated because the partial derivative is zero. This can be seen Fig. 1: for small $\beta, \mu_{y_{i j} \max }$ does not have a significant effect on the lateral force value. This is not critical because $\mu_{y_{i j} \max }$ is only needed in the transient and nonlinear area of the tire model. In the linear region, only $C_{\beta_{i j}}$ is essential. Thus, $\mu_{y_{i j} \max }$ keeps the previous value as long as the data are in the linear part.

$$
\text { if } \begin{aligned}
\lambda \geq 1 \quad: \frac{\partial F_{y_{i j}}}{\partial \mu_{y_{\max }}} & =0 \\
\frac{\partial F_{y_{i j}}}{\partial C_{\beta_{i j}}} & =\tan \left(\beta_{i j}\right) \\
\text { if } \lambda<1 \quad: \frac{\partial F_{y_{i j}}}{\partial C_{\beta_{i j}}} & =\frac{\mu_{y_{\max }}^{2} F_{z_{i j}}^{2}}{4 C_{\beta_{i j}}^{2} \tan \left(\beta_{i j}\right)} \\
\frac{\partial F_{y_{i j}}}{\partial \mu_{y_{\max }}} & =F_{z_{i j}} \operatorname{sign}\left(C_{\beta_{i j}} \tan \left(\beta_{i j}\right)\right)-\frac{F_{z_{i j}}^{2} \mu_{y_{\max }}}{2 C_{\beta_{i j}}}
\end{aligned}
$$

Therefore, for small $\beta$, it is not necessary to use the LM algorithm to estimate the parameters. Since this method does not allow to calculate $\mu_{y_{i j} \max }$, one can simply calculate $C_{\beta_{i j}}$ as the slope $a_{1}$ at the origin of $F_{y_{i j}}^{*}=a_{1} \beta_{i j}+b_{1}$ with linear least squares fitting technique. In addition, the Pacejka model does not necessarily pass through the origin unlike Dugoff model, so LM algorithm may have a lower convergence or a larger error than the affine model. This will lead to save computing time and it will allow a good initialization of $C_{\beta_{i j}}$ before the entrance into the transient regime.

\section{B. Dugoff model zone}

The optimization is performed using LM method. Let us recall that this iterative algorithm provides a solution to the problem of minimizing a multivariate function. This function is expressed as the sum of squared errors $J=$ $\sum_{n=1}^{k}\left(\hat{F}_{y}(n)-F_{y}^{*}(n)\right)^{2}$, with $k$ the number of element in the window. The LM algorithm can be seen as a combination of the steepest descent method and Gauss Newton method [34]. As output, the LM algorithm provides the cornering stiffness and maximum lateral friction coefficient.

\section{Constant zone}

In the nonlinear zone, since the Pacejka model decreases whereas Dugoff model remains constant or slightly growing, the data will not follow the model we want to fit which can cause the algorithm to converge to wrong values. In addition, updating $C_{\beta_{i j}}$ in the nonlinear zone is also risky because several values of $C_{\beta_{i j}}$ can correspond to a given $\mu_{y_{i j} \max }$. The use of LM algorithm will not be necessary and a constant model will be sufficient to calculate $\mu_{y_{i j} \max }$. As previously said, this will lead to save computing time and good initialization when entering in the transient zone. Once the constant zone identified, the constant is chosen as the mean of the lateral forces data included in the window.

\section{Determination of $\beta_{t h}$}

The purpose of estimating maximum lateral friction coefficient $\mu_{y_{i j} \text { max }}$ is to calculate the slip angle threshold value $\beta_{t h}$ for which the linear tire model is no longer valid.

The way we choose is to consider the error between the Dugoff model $F_{y \text { dug }}(2)$ for $\lambda<1$, that we call $F_{y \text { dug NL }}$ and the linear model $F_{l i n}=C_{\beta_{i j}} \beta_{i j}$. When the tire is in the linear region, the two models approximately coincide and the ratio $p=\frac{F_{\text {lin }}}{F_{y \text { dug NL }}}$ is almost equal to 1 . Thus, when $p$ 
becomes bigger than 1 , the linear model is no longer true. As the two models not exactly coincide, we consider that the slip angle threshold $\beta_{t h_{i j}}$ is reached when the ratio is higher than $p=1.05$ (experimentally determined). Thus, $\beta_{t h}$ is defined as the angle such as $F_{l i n}\left(\beta_{t h}\right)=1.05 F_{y \text { dug }}\left(\beta_{t h}\right) . \beta_{t h_{i j}}$ is obtained as following:

$$
p=\frac{C_{\beta_{i j}} \beta_{i j}}{(2-\lambda) \lambda C_{\beta_{i j}} \tan \left(\beta_{i j}\right)} \approx \frac{1}{(2-\lambda) \lambda}
$$

with $\lambda$ depending on $\beta_{i j}$ (see (3)). Solving this $2^{\text {nd }}$ order equation in $\beta_{t h_{i j}}$ gives:

$$
\begin{aligned}
\beta_{t h_{i j}} & =\frac{4 p \mu_{y_{i j} \max } F_{z_{i j}} C_{\beta_{i j}} \pm \sqrt{\Delta}}{8 C_{\beta_{i j}}^{2}} \\
\text { where } \Delta & =16 \mu_{y_{i j} \max }^{2} F_{z_{i j}}^{2} C_{\beta_{i j}}^{2}\left(p^{2}-p\right), \Delta \geq 0
\end{aligned}
$$

To be sure to maintain the tire in the linear part, we choose the smallest as the threshold :

$$
\beta_{t h_{i j}}=\frac{4 p \mu_{y_{i j} \max } F_{z_{i j}} C_{\beta_{i j}}-\sqrt{\Delta}}{8 C_{\beta_{i j}}^{2}}
$$

Remark: We could have use the definition of $\mu_{y_{i j} \max }$ (see (1)) to calculate the slip angle threshold with $F_{y_{i j} \max }=$ $C_{\beta_{i j}} \beta_{t h_{i j}}^{*}$, which gives

$$
\beta_{t h_{i j}}^{*}=\frac{\mu_{y_{\max }} F_{z_{i j}}}{C_{\beta_{i j}}}
$$

but this lead to a slip angle threshold bigger than by using (15) and the tire will already be in the nonlinear part.

\section{Simulations Results}

To evaluate the performance of the proposed algorithm, a 10 DoF vehicle model, simulated in Matlab/Simulink software is used in order to simulate the vehicle dynamics. The model takes into account both the coupling of longitudinal and lateral slip. The simulation integrates a Pacejka model for the tire described by [30]. The external disturbances such as road-bank angle, slope, and aerodynamic forces are neglected. The estimation was processed for each wheel but the results shown here will only concern the front left wheel. Simulations were performed under two different conditions, wet asphalt and snowy road. Random noise has been added to the measured and estimated lateral forces.

\section{A. Wet asphalt road}

The vehicle is moving on a lane-change trajectory as depicted in Fig. 6 at $18 \mathrm{~m} / \mathrm{s}$ on a wet asphalt such as $\mu=0.5$.

Fig. 7 shows the estimated cornering stiffness using the estimated lateral force (red) and the measured lateral force (blue). We assume that the "measured" cornering stiffness (yellow line) is the value obtained from Pacejka model under pure side slip as described in [30] and it gives us an idea of the order of magnitude. One observes that the cornering stiffness is well estimated when the measured lateral force is used, the error is globally less than $10 \%$. With the estimated lateral force, the estimation error is bigger, around $25 \%$, but it could be compensated by a robust closed loop control law. The cornering stiffness estimated with the estimated lateral force was impacted by the estimation of lateral tire force. Fig. 8 shows the error between estimated and measured left wheel lateral force. It may be noted that, the error in the estimation of lateral force occurs when the vehicle enters the left and right turn for respectively $x=0 m$ and $x=100 m$ (see Fig. 6 ) and it occurs in a short period of time as shown Fig. 9.

Fig. 10 illustrates the estimated maximum lateral friction coefficient with the measured lateral force (blue) and with the estimated lateral force (red). At the beginning of the trajectory, the vehicle is moving on a straight road, as depicted Fig. 6 and the lateral force is proportionnal to the slip angle. It corresponds, for the slip angle, to the range between 0 and $0.03 \mathrm{rad}$ in Fig. 8. In this situation, the maximum lateral friction coefficient cannot be estimated and the value is fixed to 1 . Then, the vehicle turns left, the lateral force reaches the transitional region and the estimation of $\mu_{y_{\max }}$ is processed. When the vehicle starts the right turn, the lateral force is negative. In this part of Fig. 8, the measured force has a higher magnitude than the estimated force and for $-0.05 \mathrm{rad}$ the tire is in the linear region that is why at $\mathrm{t}=11 \mathrm{~s}, \mu_{y_{\max }}=1$ when it is estimated with the measured lateral force. However, as the estimated lateral force has a lower magnitude, $\mu_{y_{\max }}$ is around 0.4 with the estimated lateral force. The difference between the 2 estimated $\mu_{y_{\max }}$ after $t=12 \mathrm{~s}$ is due to the gap between estimated and measured lateral force and since the tire returns to the linear part the $\mu_{y_{\max }}$ value is hold. Hence, the proposed estimation algorithm works well during the lane change maneuver when the lateral force is well estimated. The estimation seems to be good even if the maximum lateral friction coefficient is not precisely known (assuming it is around 0.5).

\section{B. Snowy road}

The vehicle is moving on the same trajectory as the previous simulation but at $14 \mathrm{~m} / \mathrm{s}$ on a snowy road such as $\mu=$ 0.3. Fig. 11 and Fig. 12 show respectively the results of the maximum lateral friction coefficient and cornering stiffness estimations using the estimated and measured lateral forces. Previous remarks can also be made in this situation. $\mu_{y_{\max }}$ is well estimated in both case and the error of cornering stiffness estimation is bigger with the estimated lateral force but they are both close to the expected values. Finally, Fig. 13 shows the result of slip angle threshold estimation using (15). When the affine model of the estimation algorithm can be applied (see Fig. 5), the threshold is hold at a constant value: it can be assumed that $3 \mathrm{deg}$. is generally observed for the limit of the linear region. Then, when the linear model is no longer valid, i.e. the ratio $p$ is higher than 1.05 , the slip angle threshold is updated with (15) and this value is hold when $p<1.05$. We notice in Fig. 13 (magenta dotted line) that by using (16), the slip angle threshold estimation is bigger and the tire is already in the nonlinear part while with (15) the tire has not yet reached the maximum lateral tire force available (see Fig. 14). 


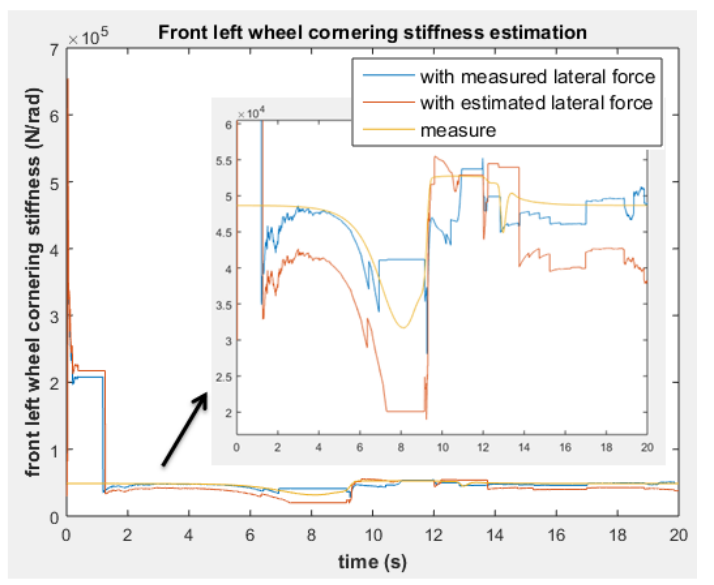

Fig. 7. Estimated and measured front left cornering stiffness for wet road

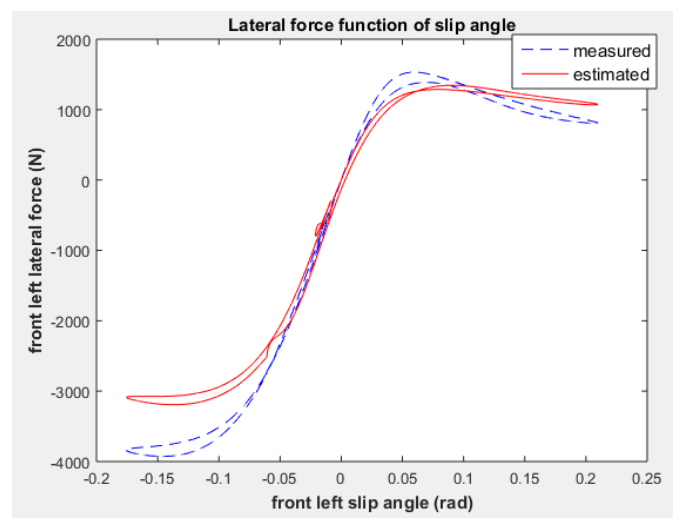

Fig. 8. Measured and estimated lateral force function of slip angle.

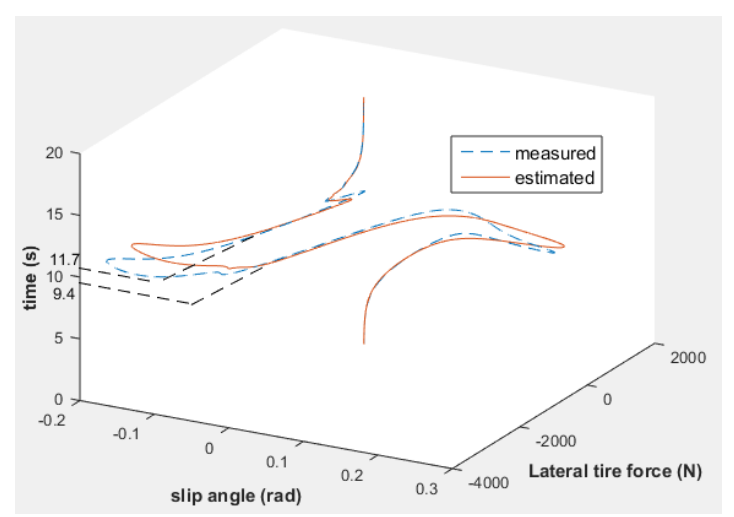

Fig. 9. Measured and estimated lateral force function of slip angle and time (black lines delimitate the short period for which the estimation error is important)

All the simulations carried out also revealed that estimating high lateral friction coefficient needs larger slip angles. In contrast, smaller slip angles are needed to have an accurate estimate of lower friction coefficient. Since the slip angle is directly related to lateral maneuvers, larger maneuvers are required to estimate high friction coefficients.

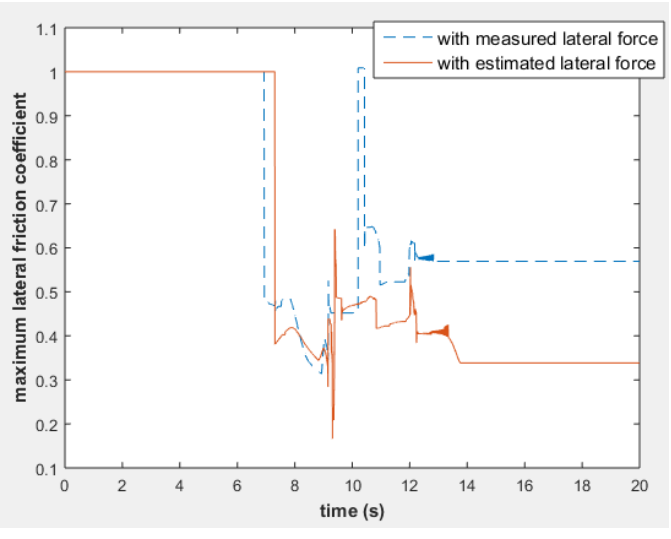

Fig. 10. Estimated $\mu_{\max }$ for wet road

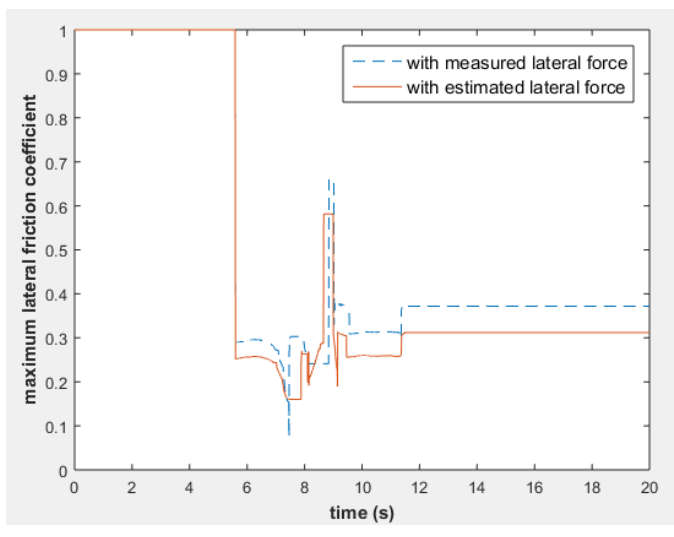

Fig. 11. Estimated $\mu_{\max }$ for snowy road

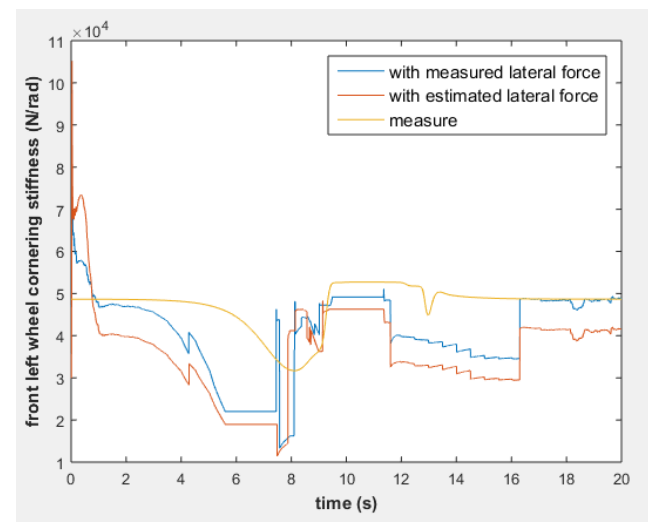

Fig. 12. Estimated and measured front left cornering stiffness for snowy road

\section{CONCLUSION}

This paper developed and investigated a new estimation method for the maximum lateral friction coefficient and the cornering stiffness which will be used in closed loop context in future developments (see [32]). For those estimations a few parameters are needed which are available through Inertial Navigation Systems. It also needs a reliable estimation of lateral tires forces. Detailed experimental results show the performance of the estimation algorithm on different road 


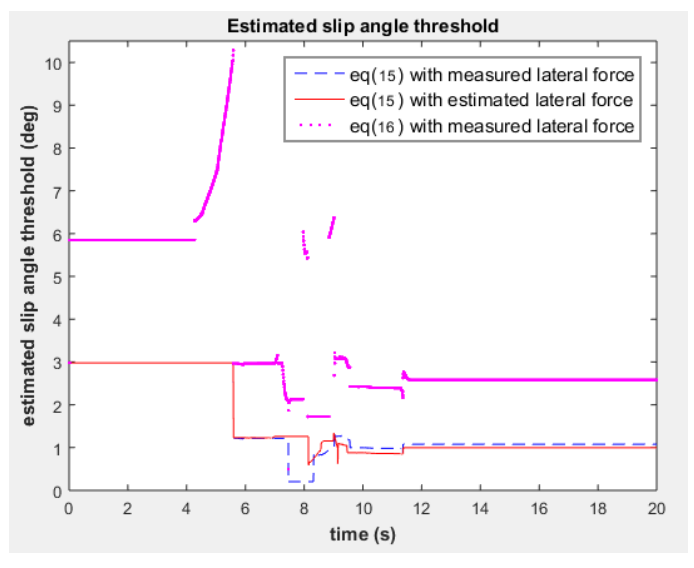

Fig. 13. Estimated slip angle threshold for snowy road

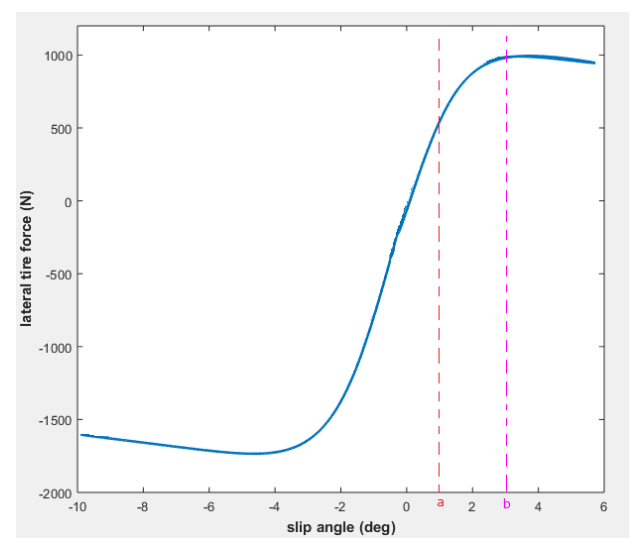

Fig. 14. Estimated slip angle threshold for snowy road : a with (15), b with (16)

conditions. Future works would also consider the comparison of this proposed algorithm to the one developped in [17].

\section{REFERENCES}

[1] R. Lenain, B. Thuilot, O. Hach, P. Martinet, High-speed mobile robot control in off-road conditions: a multi-model based adaptive approach, in IEEE International Conference on Robotics and Automation, p61436149, 2011.

[2] D. Denis, A. Nizard, B. Thuilot, R. Lenain, Slip and cornering stiffnesses observation for the stability assessment of off-road vehicles, International Conference on Mediterranean Green Energy Forum, Mar 2015, Marrakech, Morocco, MGEF, 2015.

[3] G. Baffet, A. Charara, J. Stephant, Sideslip angle, lateral tire force and road friction estimation in simulations and experiments, Proceedings of the IEEE International Conference on Control Applications, Munich, Germany, October 4-6, 2006.

[4] U. Eichhorn and J. Roth, Prediction and monitoring of tyre/road friction, Proc. FZSZTA, London, June 1992.

[5] B. Breuler, U. Eichhorn, J. Roth, Measurement of tyre/road friction ahead of the car and inside the tyre, In Proceedings of the international symposium on AVEC, p347-353, 1992.

[6] Th. Bachmann, The importance of the integration of road, tyre, and vehicle technologies, FISITA XXth World Congress, Montreal, Canada, September 1995.

[7] M.R. Uchanski, Road Friction Estimation for Automobiles Using Digital Signal Processing Methods,PhD Thesis, University of California, Berkeley, 2001.

[8] A. Andersson, Road-Tire Friction Estimation for AFS Vehicle Control, Master Thesis, August 2005.
[9] L. Ray, Nonlinear Tire Force Estimation and Road Friction Identification, Simulation and Experiments, Automatica, 33, no. 10, pp.18191833, 1997.

[10] E Gustafsson, Slip-based estimation of tire-road friction, Automatica, vol. 33, pp. 1087-1099, 1997.

[11] R. Castro, R. Ara, Real-time Estimation of Tire-Road Friction Peak with Optimal Linear Parameterization, In IET Control Theory and Applications, September 2012.

[12] M. Tanelli, L. Piroddi, S. Savaresi, Real-time identification of tireroad friction conditions, in IET Control Theory and Applications, July 2008.

[13] A. Senatore, M. Sharifzadeh, Estimation of tyre-road friction during ABS braking for snow and ice conditions,6 Workshop AIT Tribologia e Industria, Torino, April 2018.

[14] M. Sharifzadeh, A. Akbari, F. Timpone, R. Daryani, Vehicle tyre/road interaction modeling and identification of its parameters using realtime trust-region methods, IFAC, 2016.

[15] C. Ahn, H. Peng, E. Tseng, Estimation of Road Friction for Enhanced Active Safety Systems: Algebraic Approach,American Control Conference Hyatt Regency Riverfront, St. Louis, MO, USA, 2009.

[16] C. Hu, R. Wang, Z. Wang, M. Chadli, F. Yan, Integrated Optimal Dynamics Control of 4WS4WD Electric Ground Vehicles with TireRoad Frictional Coefficient Estimation, American Control Conference Palmer House Hilton, Chicago, IL, USA, 2015.

[17] J. Hahn, R. Rajamani, GPS-based real-time identification of tireroad friction coefficient, In IEEE Transactions on Control Systems Technology, Vol.10, May 2012.

[18] M. Doumiati, A. Charara, A. Victorino, D. Lechner, Vehicle Dynamics Estimation using Kalman Filtering, Series Editor, Bernard Dubuisson, 2012.

[19] G. Baffet, A. Charara and J. Stephant, Sideslip angle, lateral tire force and road friction estimation in simulations and experiments, IEEE International Conference on Control Applications, Munich, Germany, 2006.

[20] G.Baffet, A. Charara, G.Dherbomez, An observer of tire-road forces and friction for active security vehicle systems, IEEE/ASME Trans. Mechatron., vol. 12, no. 6, pp. 651?661, Dec. 2007.

[21] M. Doumiati, A. Victorino, A. Charara, D. Lechner, A method to estimate the lateral tire force and the sideslip angle of a vehicle: Experimental validation, American Control Conference Marriott Waterfront, Baltimore, MD, USA June 30-July 02, 2010.

[22] J. Dakhlallah, S. Glaser, S. Mammar, Y. Sebsadji, Tire-road forces estimation using extended Kalman filter and sideslip angle evaluation, American Control Conference, Seattle,Washington, U.S.A, June 2008.

[23] M. Doumiati, A. Charara, A. Victorino, D. Lechner, Vehicle Dynamics Estimation using Kalman Filtering,Great Britain and the United States by ISTE Ltd and JohnWiley \& Sons, Inc.

[24] M. Doumiati, A. Victorino, A. Charara, D. Lechner, Abstract?Extensive Estimation of Vehicle Lateral Tire-Road Forces: A Comparison between Extended and Unscented Kalman Filtering

[25] M. Doumiati, G. Baffet, D Lechner, A. Victorino, A. Charara, Embedded estimation of the tire/road forces and validation in a laboratory vehicle, Proceedings of 9th International Symposium on Advanced Vehicle Control, Kobe Japan

[26] G. Baffet, A. Charara, D. Lechner, Estimation of vehicle sideslip, tire force and wheel cornering stiffness, Control Engineering Practice, Vol 17, Issue 11, November 2009.

[27] N.K. M'Sirdi, A. Rabhi, N. Zbiri, Y. Delanne,VRIM: Vehicle - Road Interaction Modelling for Estimation of Contact Forces

[28] G. Baffet, A. Charara, D. Lechner, Estimation of Tire-Road Forces and Vehicle Sideslip Angle, Advances in Robotics, Automation and Control, October 2008, I-Tech, Vienna, Austria

[29] J. Yang, W. Chen, Y. Wang, Estimate lateral tire force based on yaw moment without using tire model

[30] H.B. Pacejka, Tyre and Vehicle Dynamics. Butterworth-Heinemann

[31] JVillagra, B. D'Andréa-Novel, M. Fliess, H. Mounier, A diagnosisbased approach for tire-road forces and maximum friction estimation. Control Engineering Practice, Elsevier, 2011, 19 (2), pp.174-184.

[32] L. Li, B. D'Andréa-Novel, S. Thorel, A coupled nonlinear controller for two steering axles vehicles, internal report.

[33] R. Rajamani, Vehicle Dynamics and Control, Springer, 2012.

[34] K. Madsen, H.B. Nielsen, O. Tingleff, Methods for Non-Linear Least Squares Problems (2nd ed.), 2004 